\title{
RELATION BETWEEN SALIVARY CORTISOL AND ALPHA AMYLASE LEVELS AND ANXIETY IN EGYPTIAN PATIENTS WITH MINOR RECURRENT APHTHOUS STOMATITIS
}

\author{
Mai Zakaria* and Ragaa Hosny**
}

\begin{abstract}
Background/Aim: Recurrent aphthous stomatitis (RAS) is the most prevalent ulcerative disorder of the oral mucous membrane. The aim of the present contemplate is to assess salivary cortisol (SC) and salivary alpha amylase enzyme (SAA) levels in relation to anxiety in Egyptian patients with minor recurrent aphthous stomatitis.

Subjects and Methods: patients with minor RAS as a study group (RAS group, $\mathrm{n}=35$ ) and healthy subjects as a control (control group, $\mathrm{n}=35$ ) who were matching regarding age, sex, and socioeconomic state were included in this study. SC and SAA were assessed in all participants using enzyme-linked immunosorbent assay (ELISA) and kinetic enzyme assay respectively. Hamilton's anxiety scale (HAS) has been used for evaluation of anxiety level in both study groups.

Results: A significantly high mean SC $(1.7 \pm 0.34 \mu \mathrm{g} / \mathrm{dl})$, SAA $(137.87 \pm 47.16 \mathrm{U} / \mathrm{ml})$ levels and anxiety score $(26.7 \pm 2.21)$ were recorded in RAS group $(\mathrm{p}=0.00,0.02,0.00)$ respectively in comparison to control group.
\end{abstract}

Conclusion: Anxiety may have a pivotal role in etiopathogensis of RAS hence; Psychological management should be considered in RAS treatment.

KEYWORDS: Recurrent aphthous stomatitis, saliva, cortisol, alpha amylase, anxiety.

\section{INTRODUCTION}

Recurrent aphthous stomatitis (RAS) is the most prevalent ulcer affecting oral mucous membrane where it involves up to $25 \%$ of general population ${ }^{[1]}$. It occurs as painful, shallow, round or oval oral ulcers encompassed by erythematous ring and associated with recurrence. RAS has three clinical types: minor RAS, major RAS, and herpetiform ulcerations ${ }^{[2]}$.

The most widely recognized type of RAS is the minor form; involving nearly $80 \%$ of RAS patients. The ulcer size of the minor form is less than $1 \mathrm{~cm}$

\footnotetext{
* Lecturer, Oral Medicine and Periodontology Department, Faculty of Dentistry, Cairo University, Egypt
}

** Professor, Biooncology Department, National Cancer Institute, Cairo University, Egypt 
in diameter and not associated with scarring. The ulcers of the major form are larger than $1 \mathrm{~cm}$ in diameter, persist for more time and the healing is frequent with scars formation. Herpetiform type shows as clusters of many tiny ulcers all through the oral mucous membrane ${ }^{[3,4]}$. RAS is painful and obstacles with every day oral activity for example, eating, talking and deglutition, subsequently diminishing personal satisfaction ${ }^{[5]}$.

The exact cause of RAS is unclear, yet multiple local, systemic, immunological, hereditary, allergic, nutritional and microbial variables were involved to be etiological factors ${ }^{[6]}$. Additionally, RAS has been proposed to be associated with stress and anxiety; where they could assume a part in the beginning and repeat of RAS sores ${ }^{[7,8]}$. The high recurrence and diminished personal satisfaction produced by RAS have brought about a lot of research into the etiology and proficient treatment of this sickness ${ }^{[9]}$.

It has been postulated that one of the most etiologic factor of RAS is stress with its assumed impacts on the immune system components ${ }^{[10]}$. Previous investigations showed expanded recurrence of oral ulcer sign in populations with serious stresses $^{[11]}$.

Cortisol, additionally known as stress hormone; with its assessments in saliva became very important for anxiety and stress evaluation in studies ${ }^{[12]}$. Salivary cortisol (SC) may really give a superior assessment of stress reaction in comparison to serum cortisol because it estimates the unbound cortisol level more precisely ${ }^{[13]}$.

The salivary alpha amylase enzyme (SAA) is delivered by the salivary glands and it starts the processing of larger molecules, for example, starches into simpler ones ${ }^{[14]}$. The discharge of SAA is controlled by sympathetic autonomic nervous system (SANS) activity which significantly manages the stress psychobiology ${ }^{[15]}$. Trails demonstrated increased levels of SAA in humans with physical and psychological stress ${ }^{[16]}$. Consequently, the SAA has progress toward becoming a marker of stress and anxiety ${ }^{[17]}$.

Recently, both SC and SAA have been utilized as biomarkers of stress ${ }^{[18,19]}$. Because the etiology of RAS still is unknown, the commonly used treatment remains inadequate ${ }^{[9]}$. Several investigations have revealed a connection amongst RAS and different causes; however the outcomes are conflicting. ${ }^{[10]}$

Accordingly, the present study was conducted to assess SC and SAA levels in relation to anxiety in Egyptian patients with minor RAS.

\section{SUBJECTS AND METHODS}

\section{Subjects \& Study design}

This study was conducted in the Department of Oral Medicine and Periodontology, Faculty of Dentistry, Cairo University, after ethics committee approval and registered in code no 17/ 10/ 13 . The study was carried out according to the ethical guidelines of the World Medical Association (Declaration of Helsinki) for studies involving human subjects. The study enrolled 35 patients with minor RAS as study group (RAS group) along with 35 healthy subjects who were matching regarding age, sex and socio-economic status (SES) as a control (control group) without any history of RAS, after explanation of the study protocol a written informed consent was obtained from all participants. The SES was determined using Modified Kuppuswamy's scale ${ }^{[20]}$.

Medical data were collected from all the study participants according to the Modified Cornel Medical Index questionnaire ${ }^{[21]}$. The diagnosis of RAS was done according to the patients' history and the clinical features of the lesions ${ }^{[22]}$. The inclusion criteria for selection of the patients were as follows: active minor RAS, aged (18 - 30) years, nonsmokers, and at least 2 years of RAS history. The exclusion criteria were periodontal disease, pregnancy, lactation, fever, history of diabetes mellitus, 
smoking, hypertension, nutritional deficiency, traumatic ulcers, HIV infection, Behcet's disease, Crohn's disease, Coeliac disease, Addison's disease, and infectious disease. Also, subjects with history of drug intake like steroids, oral contraceptive, nicorandil, anti-inflammatory drugs, antidepressants, beta-blockers, anxiolytics, and oral nicotine replacement therapy were omitted. In addition, we excluded subjects on any other drug that is known to disturb the autonomic function or cortisol and amylase levels.

\section{Basis of sample size calculation}

\section{Salivary cortisol}

According to Nadendla et al ${ }^{[23]}$, the mean SC levels were $(1.73 \pm 0.319) \mu \mathrm{g} / \mathrm{dl}$ in RAS patients and $(0.504 \pm 0.129) \mu \mathrm{g} / \mathrm{dl}$ in controls. Sample size was calculated as $n=28$ for each group.

\section{Salivary alpha-amylase}

According to Cardoso et al ${ }^{[24]}$, median SAA (U/ $\mathrm{ml})$ was $42.72(25.18-75.55)$ in controls, 45.50 (35.68-97.47) in RAS patients. The mean was calculated as 46.54 in controls and 56.04 in RAS patients using the formula below:

We found that a simple formula (5):

$$
\bar{X}=\frac{a+2 \mathrm{~m}+\boldsymbol{b}}{4}
$$

can be used to estimate the mean using the values of the median $(m)$, low and high end of the range (a and $b$, respectively).

Sample size was calculated as $n=35$ for each group.

Accordingly, the sample size for each group in respect to both SC and SAA was 35 participants.

\section{Salivary Sample Collection and Questionnaire Survey}

Salivary sample collection from both study groups was performed using whole unstimulated technique between (8:00 - 10:00) AM to avoid diurnal variations of salivary proteins ${ }^{[25]}$. All the participants were required not to eat, drink, or do oral hygiene measures nor put any substance in the mouth (including brushing teeth), do physical exercises, or use drugs or cosmetics in lips for at least $1 \mathrm{~h}$ before the sample collection to decrease its impurities ${ }^{[26]}$. Prior to collection of salivary samples, they rinsed the mouth with water only without using any substance to enhance salivary secretion. They were instructed to expectorate their saliva into a sterile disposable plastic tube once per minute until $7 \mathrm{ml}$ was collected. Then the samples were sent immediately to the clinical laboratory, Department of Biooncology, National Cancer Institute, Cairo University.

After salivary samples collection, the subjects were asked to answer questionnaire, namely Hamilton's anxiety scale (HAS) that measures of overall anxiety, psychic anxiety (mental agitation and psychological distress) and somatic anxiety (physical complaints related to anxiety) for psychological evaluation. This scale is composed of 14 questions, where seven indicate the psychic and the other ones indicate the somatic anxiety. There are five point scales for each of the 14 question from 0 to 4 . The total anxiety score ranges from 0 to 56 , where $<17$ addresses mild severity, 18-24 mild to moderate severity and 25-30 moderate to severe ${ }^{[23]}$.

\section{Salivary Protein Analysis}

The collected salivary samples were immediately mixed with protease inhibitors to prevent degradation of the proteins and centrifuged at $3000 \mathrm{rpm}$ for $5 \mathrm{~min}$, resulting in a clear supernatant. Then, the samples were stored at $-20^{\circ} \mathrm{C}$ until shortly before the assay. During the assay, the frozen samples were thawed at $37^{\circ} \mathrm{C}$, the supernatants fluids which were obtained then were used for determinations of SC and SAA levels. Quantitative determination of SC $(\mu \mathrm{g} / \mathrm{dl})$ was performed by using an enzyme-linked 
immunosorbent assay (ELSA) kit $\left(\right.$ Salimetrics $\left.^{\mathrm{R}}\right)$. The Kinetic Enzyme Assay Kit (KEA) (Salimetrics ${ }^{\odot}$ ) was used for the analysis of SAA (expressed as $\mathrm{U} / \mathrm{ml})$.

\section{Statistical analysis}

Statistical analysis was then performed using a commercially available software program (SPSS 18; SPSS, Chicago, IL, USA).

Values were presented as mean and standard deviation (SD) and $95 \%$ confidence interval values. Data were explored for normality using Kolmogorov-Smirnov test of normality. The results of Kolmogorov-Smirnov test indicated that most of data were normally distributed (parametric data), so unpaired (independent) $t$ test was used to compare control and RAS groups.

Pearson correlation test was used for SC, SAA and anxiety score correlation. The Pearson correlation coefficient was used to measure the strength of a linear association between two variables, as follows: Exactly -1 represents perfect negative linear relationship. 0 represents no linear relationship. Exactly +1 represents a perfect uphill (positive) linear relationship. The level of significance was set at $\mathrm{P}<0.05$.

\section{RESULTS}

Each group in this study consisted of 35 subjects (50\% males and 50\% females), with no difference in gender distribution. The mean age in RAS group was $(23.2 \pm 3.97)$ years, while mean age in control group was $(23 \pm 4.03)$ years, with no significant difference $(\mathrm{p}=0.91)$ as presented in table 1 .

A significantly higher mean SC $(1.7 \pm 0.34 \mu \mathrm{g} /$ $\mathrm{dl})$, SAA $(137.87 \pm 47.16 \mathrm{U} / \mathrm{ml})$ and anxiety score $(26.7 \pm 2.21)$ were noted in RAS group $(\mathrm{p}=0.00$, $0.02,0.00)$ respectively in comparison to control group where mean $\mathrm{SC}(0.47 \pm 0.05 \mu \mathrm{g} / \mathrm{dl}), \mathrm{SAA}$ $(105.31 \pm 6.35 \mathrm{U} / \mathrm{ml})$ and anxiety score $(15.5 \pm 2.42)$ were noted in healthy subjects (Table 1, Fig. 1-3).

TABLE (1) Age, salivary cortisol, salivary alpha amylase levels and anxiety scores in study groups

\begin{tabular}{|c|c|c|c|c|c|c|c|c|c|}
\hline & \multirow{2}{*}{ Groups } & \multirow{2}{*}{ Mean } & \multirow{2}{*}{ Std. Dev } & \multirow{2}{*}{$\begin{array}{c}\text { Mean } \\
\text { Difference }\end{array}$} & \multirow{2}{*}{$\begin{array}{l}\text { Std. Error } \\
\text { Difference }\end{array}$} & \multicolumn{2}{|c|}{ 95\% C.I. diff. } & \multirow{2}{*}{$\mathrm{T}$} & \multirow{2}{*}{$\mathrm{p}$} \\
\hline & & & & & & Lower & Upper & & \\
\hline \multirow{2}{*}{ Age } & RAS & 23.20 & 3.97 & \multirow{2}{*}{0.20} & \multirow{2}{*}{1.79} & \multirow{2}{*}{-3.56} & \multirow{2}{*}{3.96} & \multirow{2}{*}{0.11} & \multirow{2}{*}{$0.91^{\mathrm{ns}}$} \\
\hline & Control & 23.00 & 4.03 & & & & & & \\
\hline \multirow{2}{*}{$\begin{array}{l}\text { Salivary cortisol } \\
\qquad(\mu \mathrm{g} / \mathrm{dl})\end{array}$} & RAS & 1.70 & 0.34 & \multirow{2}{*}{1.23} & \multirow{2}{*}{0.11} & \multirow{2}{*}{0.99} & \multirow{2}{*}{1.47} & \multirow{2}{*}{11.34} & \multirow{2}{*}{$0.00^{*}$} \\
\hline & Control & 0.47 & 0.05 & & & & & & \\
\hline \multirow{2}{*}{$\begin{array}{l}\text { Salivary alpha } \\
\text { amylase }(\mathrm{U} / \mathrm{ml})\end{array}$} & RAS & 137.87 & 37.16 & \multirow{2}{*}{32.56} & \multirow{2}{*}{11.92} & \multirow{2}{*}{5.82} & \multirow{2}{*}{59.31} & \multirow{2}{*}{2.73} & \multirow{2}{*}{$0.02 *$} \\
\hline & Control & 105.31 & 6.35 & & & & & & \\
\hline \multirow{2}{*}{ Anxiety score } & RAS & 26.70 & 2.21 & \multirow{2}{*}{11.20} & \multirow{2}{*}{1.04} & \multirow{2}{*}{9.02} & \multirow{2}{*}{13.38} & \multirow{2}{*}{10.81} & \multirow{2}{*}{$0.00^{*}$} \\
\hline & Control & 15.50 & 2.42 & & & & & & \\
\hline
\end{tabular}

95\% C.I. diff.= 95\% Confidence Interval of Difference

Significance level $p<0.05$, *significant, $n s=n o n$-significant

\footnotetext{
${ }^{\mathrm{R}} 101$ Innovation Boulevard, State College, PA

- 101 Innovation Boulevard, State College, PA
} 


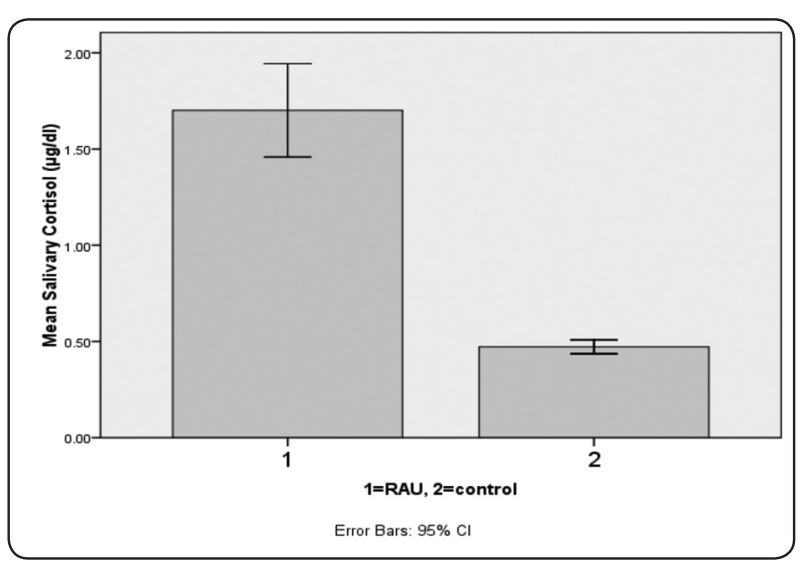

Fig. (1) Bar chart of mean salivary cortisol level in RAS group (group 1) and control group (group 2)

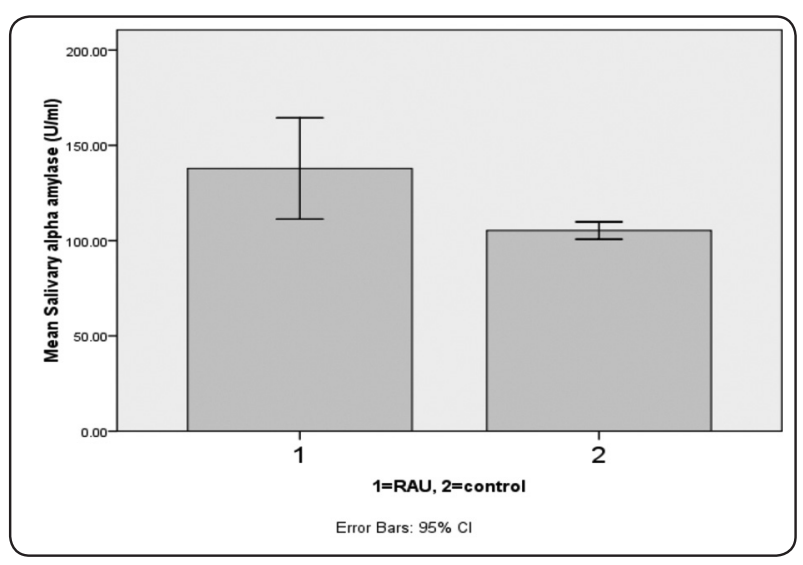

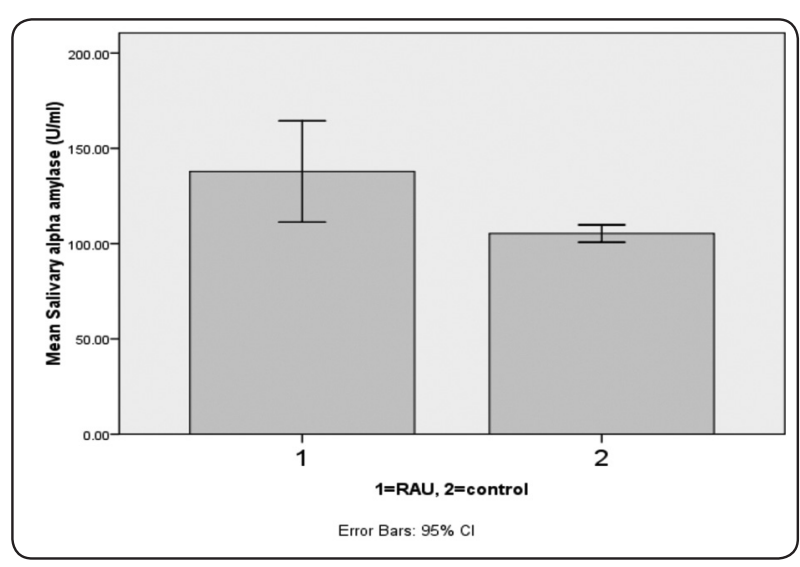

Fig. (2) Bar chart of mean salivary alpha amylase level in RAS group (group 1) and control group (group 2)

Fig. (3) Bar chart of mean anxiety score in RAS group (group 1) and control group (group 2)

A strong positive significant correlation was noted between SC, SAA and anxiety score (Table 2, Fig. 4-6).

TABLE (2) Correlation between salivary cortisol, salivary alpha amylase levels and anxiety score (Pearson Correlation test)

\begin{tabular}{|c|c|c|c|c|}
\hline & & Salivary cortisol & Salivary alpha amylase & Anxiety score \\
\hline \multirow[t]{3}{*}{ Salivary cortisol } & Pearson Correlation & ---- & $.733^{* *}$ & $.962^{* *}$ \\
\hline & Sig. (2-tailed) & & .000 & .000 \\
\hline & $\mathrm{N}$ & - & Strong +VE & Strong +VE \\
\hline \multirow[t]{3}{*}{ Salivary alpha amylase } & Pearson Correlation & $.733^{* *}$ & ---- & $.721^{* *}$ \\
\hline & Sig. (2-tailed) & .000 & & .000 \\
\hline & $\mathrm{N}$ & Strong +VE & & Strong +VE \\
\hline \multirow[t]{3}{*}{ Anxiety score } & Pearson Correlation & $.962^{* *}$ & $.721^{* * *}$ & ---- \\
\hline & Sig. (2-tailed) & .000 & .000 & \\
\hline & $\mathrm{N}$ & Strong +VE & Strong +VE & \\
\hline \multicolumn{3}{|c|}{ **. Correlation is significant at the 0.01 level (2-tailed). } & & \\
\hline
\end{tabular}




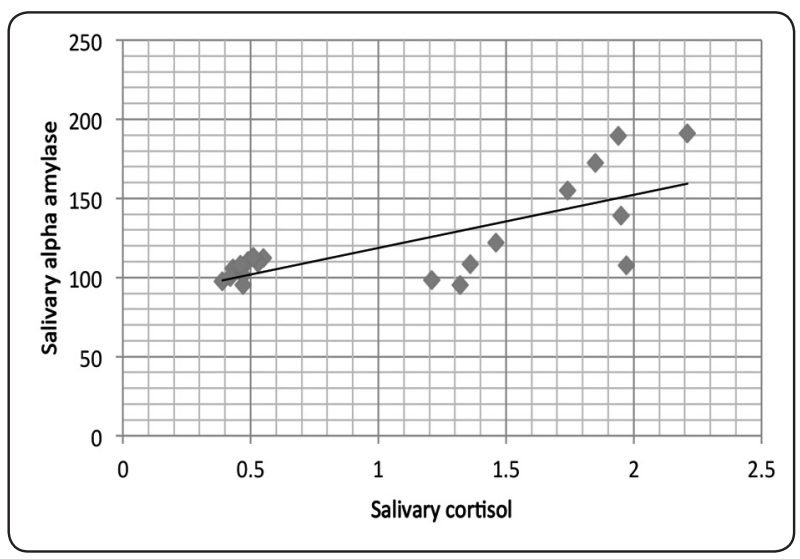

Fig. (4) Scatter plot showing correlation between salivary cortisol and salivary alpha amylase levels

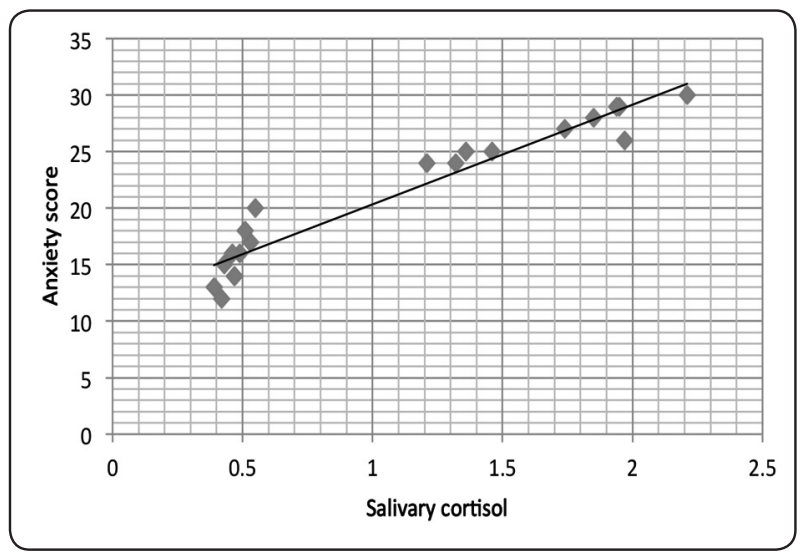

Fig. (5) Scatter plot showing correlation between salivary cortisol level and anxiety score

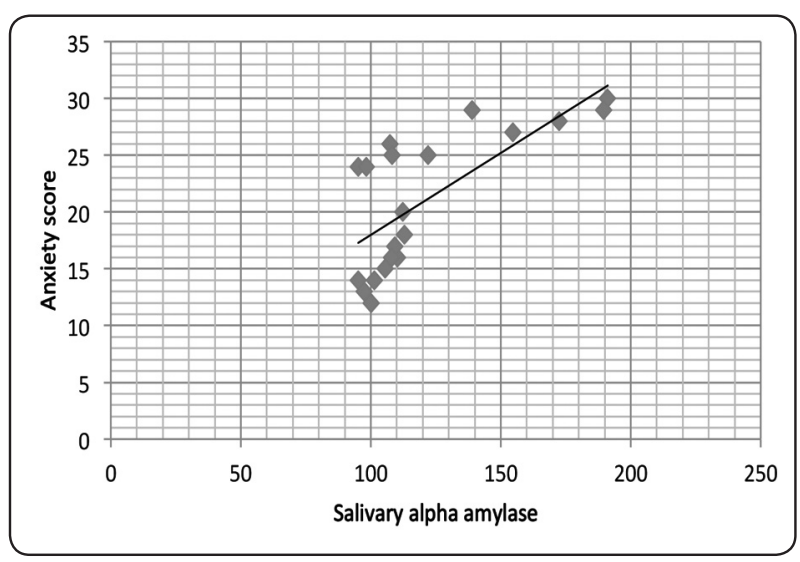

Fig. (6) Scatter plot showing correlation between salivary alpha amylase level and anxiety score

\section{DISCUSSION}

The RAS is a common painful disorder affecting oral mucous membrane in the form of single or multiple ulcerative lesions characterized by recurrence [22]. These lesions induce pain and may interfere with eating, speaking, and oral cleanliness, prompting more serious effect on patient's life quality ${ }^{[24]}$.

RAS is believed to be a stress related reaction. The sympatho-adrenal system or the hypothalamopituitary axis that become empowered during stress time doesn't succeed to return back to the basal levels in RAS. In the periods of stress, the body's immune pathways are hampered to a specific degree. Stress is well known to influence different immune system mechanisms such as phagocytosis of macrophages, cytokines secretion, and immunoglobulins synthesis. Furthermore, the production, dispersion and action of lymphocytes and natural killer cells are significantly affected. The stress hormones and biomarkers increased as a result to that ${ }^{[27,28]}$.

Collection of salivary samples is simple, cheap, stress free and noninvasive procedures and needs little preparations ${ }^{[29]}$. HAS is one of the widely used scales in the clinical studies being reliable and time saving; so it has been utilized in this study to evaluate the anxiety severity. Hence, the present contemplate was carried out to assess the relation between SC and SAA as stress biomarkers and anxiety in patients with RAS.

This study results found that a significantly higher mean of anxiety score was recorded in patients with RAS in comparison to controls. This coincides with the results revealed by Nadendla et al. ${ }^{[23]}$ who used HAS for the assessment of the anxiety in RAS patients which is the same scale used in this study. Despite different scales were used by AlbanidouFarmaki et al. ${ }^{[10]}$, Gallo et al. ${ }^{[9]}$, Bilal et al. ${ }^{[27]}$, and Cardoso et al ${ }^{[24]}$ who used Spielberger's State- Trait Anxiety Inventory (STAI), Symptoms of Stress List (SSL), STAI; Beck Depression Inventory (BDI), and Lipp's Inventory of Stress Symptoms; DDI 
respectively similar findings were found by them regarding the evaluation of anxiety in RAS patients. But this was not in accordance with findings previously showed by Picek et al. ${ }^{[8]}$, who revealed no significant differences between the controls and RAS patients in the acute state in relation to anxiety therapy or state sores. This may be due to that development of RAS sores makes patients more anxious rather than that psychological diseases leading to their appearance.

The present contemplate results revealed that significantly higher mean of SC level in RAS patients was recorded in comparison to control participants. This agrees with the findings previously revealed by McCartan et al. ${ }^{[30]}$, Albanidou-Farmaki et al. ${ }^{[10]}$, and Nadendla et al. ${ }^{[23]}$. While this is against the results of the study conducted by Kunikullaya et al. ${ }^{[28]}$ which showed no statistically significant difference in SC between RAS group and control one. This may be due to different technique used for salivary samples collection.

In addition, the outcomes of this study showed that significantly high difference in the mean of SAA levels between the patients having RAS and the participants of the control group. Dissimilar findings were reported by Cardoso et $\mathrm{al}^{[24]}$ and Kunikullaya et al ${ }^{[28]}$; this may be due to different range of age (19-69) years in the participants' inclusion criteria which may affect SAA secretion and different utilized technique in salivary samples collection respectively.

Cortisol secretion is controlled by the hypothalamo-pituitary-adrenal axis and this is alongside adrenocorticotropic hormone, changes in relation to acute and chronic stress. In case of stress the increase in the level of cortisol is preserved and it is straight forwardly corresponding to the seriousness of stress. Stress and anxiety are thought to hasten the event of RAS with rises in SC levels ${ }^{[9,30]}$.

The activity of salivary gland is estimated as a circuitous estimation of sympatho-adrenomedullary system as a result of stress. In the recent years some studies have utilized SAA as a dependent stress biomarker ${ }^{[31,32]}$. SAA is exceptional, because it is manufactured by salivary glands acini; rather than being transported by blood stream into saliva and its discharge is autonomous of salivary flow rate ${ }^{[33]}$. It associates well with the sympathetic activity and level of norepinephrine ${ }^{[34]}$.

The oral mucosa is protected by saliva because of existence of lysozymes and secretory immunoglobulin A. SAA is the salivary catalyst that starts starch digestion. But the discharged salivary catalysts without the presence of food in the oral cavity can prompt unwanted changes in the mucosa. It could be assumed that even a slight rise of this enzyme alongside dysregulation in the defensive immune components could trigger the occasion of RAS. In addition, supported ascent in cortisol levels with amylase could induce the pathophysiological events for ulcer proceeding ${ }^{[28]}$.

\section{CONCLUSION}

It could be concluded that RAS patients have high anxiety scores, SC and SAA levels where they could be used as stress biomarkers. Accordingly, anxiety may have a pivotal role in etiopathogensis of RAS hence; Psychological management should be considered in RAS treatment. Within the limitation of the current study, larger sample size should be included to accurately assess SC and SAA levels in relation to anxiety among Egyptian patients with minor recurrent aphthous stomatitis.

\section{REFERENCES}

1. Akintoye SO, Greenberg MS. Recurrent aphthous stomatitis. Dent Clin N Am. 2014; (58) 281- 297.

2. Khabbazi A, Ghorbanihaghjo A, Farahnoosh Fanood F, Sousan Kolahi S, Hajialiloo M, Rashtchizadeh N. A comparative study of vitamin D serum levels in patients with recurrent aphthous stomatitis. The Egyptian Rheumatologist. 2015; 37, 133-137. 
3. Natah SS, Konttinen YT, Enattah NS, Ashammakhi N, Sharkey KA, Ha yrinen-Immonen R. Recurrent aphthous ulcers today: a review of the growing knowledge. Int $\mathrm{J}$ Oral Maxillofac Surg. 2004; 33:221e34.

4. Chavan M, Jain H, Diwan N, Khedkar S, Shete A, Durkar S. Recurrent aphthous stomatitis: a review. J Oral Pathol Med 2012; 41:577e83.

5. Krisdapong S, Sheiham A, Tsakos G. Impacts of recurrentaphthous stomatitis on quality of life of 12- and 15-year old Thai children. Qual Life Res. 2012; 21: 71-76.

6. Chavan M, Jain H, Diwan N, Khedkar S, Shete A, Durkar S. Recurrent aphthous stomatitis: a review. J Oral Pathol Med. 2012; 41: 577- 583.

7. Huling LB, Baccaglini L, Choquette L, Feinn RS, Lalla RV. Effect of stressful life events on the onset and duration of recurrent aphthous stomatitis. J Oral Pathol Med. 2012; 41: 149-152.

8. Picek P, Buljan D, Rogulj AA et al Psychological status and recurrent aphthous ulceration. Coll Antropol.2012; 36:157-159.

9. Gallo Cde B, Mimura MA, Sugaya NN. Psychological stress and recurrent aphthous stomatitis. Clinics (Sao Paulo) 2009; 64:645- 8 .

10. Albanidou-Farmaki E, Poulopoulos AK, Epivatianos A, Farmakis K, Karamouzis M, Antoniades D. Increased anxiety level and high salivary and serum cortisol concentrations in patients with recurrent aphthous stomatitis. Tohoku J Exp Med. 2008; 214: 291- 6.

11. Soto Araya M, Rojas Alcayaga G, Esguep A. Association between psychological disorders and the presence of Oral lichen planus, Burning mouth syndrome and Recurrent aphthous stomatitis. Med Oral. 2004; 9 :1-7.

12. Koray M, Dülger O, Ak G, Horasanli S, Uçok A, Tanyeri $\mathrm{H}$, et al. The evaluation of anxiety and salivary cortisol levels in patients with oral lichen planus. Oral Dis. 2003; 9: 298- 301.

13. Safarzadeh E, Mostafavi F, Haghi-Ashtiani MT. Determination of salivary cortisol in healthy children and adolescents. Acta Med Iran. 2005; 43: 32- 6.

14. Sampaio N, Mello S, and Alves C. Dental caries-associated risk factors and type 1 diabetes mellitus. Pediatric Endocrinology, Diabetes and Metabolism, 2011; 17:152-157.

15. Granger D A, Kivlighan K T, El-Sheikh M, Gordis E B, and Stroud L R, "Salivary alpha-amylase in biobehavioral research: recent developments and applications," Annals of the New York Academy of Sciences. 2007; 10 (98): 122-144.

16. Kang Y. "Psychological stress-induced changes in salivary alpha-amylase and adrenergic activity," Nursing and Health Sciences. 2010; 12: 477- 484.

17. Nater U M and Rohleder N. "Salivary alpha-amylase as a noninvasive biomarker for the sympathetic nervous system: current state of research," Psychoneuroendocrinology. 2009; 34 (4):486- 496.

18. Maruyama, Y., Kawano, A., Okamoto, S., Ando, T., Ishitobi, Y., Tanaka, Y., Inoue, A., Imanaga, J., Kanehisa, M., Higuma, H., Ninomiya, T., Tsuru, J., Hanada, H. and Akiyoshi, J. Differences in salivary alpha-amylase and cortisol responsiveness following exposure to electrical stimulation versus the Trier social stress tests. PLoS One. 2013; 7: e39375.

19. Tamura, A., Maruyama, Y., Ishitobi, Y., Kawano, A., Ando, T.,Ikeda, R., Inoue, A., Imanaga, J., Okamoto, S., Kanehisa, M., Ninomiya, T., Tanaka, Y., Tsuru, J. and Akiyoshi, J. Salivary alpha amylase and cortisol responsiveness following electrical stimulation stress in patients with the generalized type of social anxiety disorder. Pharmacopsychiatry 2013; 46: 225-260.

20. Singh T, Sharma S, Nagesh S. Socio-economic status scales updated for 2017. Int J Res Med Sci. 2017; 5(7):3264-3267

21. Brightman VJ. Red and white lesions of the oral mucosa.

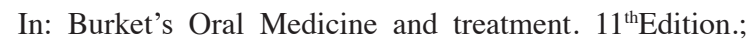
Eds: Greenberg M and Glick M, 2003

22. Chattopadhyay A and Shetty V. Recurrent aphthous stomatitis. Otolaryngologic Clinics of North America, 2011; 44 (1): 79-88.

23. Nadendla K, Venkateswarlu Meduri V, Paramkusam G, and Pachava R. Relationship of salivary cortisol and anxiety in recurrent aphthous stomatitis. Indian J Endocrinol Metab. 2015; 19(1): 56-59.

24. Cardoso JU, Junior AS, Nunes ML, Figueiredo MA,Karen Cherubini K, and Salum FG. Salivary Alpha-Amylase Enzyme, Psychological Disorders, and Life Quality in Patients with Recurrent Aphthous Stomatitis. International Journal of Dentistry. 2017; 3:1-6.

25. Schubert C, Lampe A, Geser W, et al. Daily psychosocial stressors and cyclic response patterns in urine cortisol and neopterin in a patient with systemic lupus erythematosus. Psychoneuroendocrinology.2003; 28:459- 473. 
26. Navazesh M. Methods for collecting saliva. Ann N Y Acad Sci.1993; 694:72-77.

27. Bilal N, Fatih Karakus M, Varkal MD, Boztepe OF, Bilal B, et al. An Assessment of the Levels of Anxiety and Depression in Patients with Recurrent Aphthous Stomatitis. Arch Otolaryngol Rhinol. 2016; 2(1): 001-005.

28. Kunikullaya U, Kumar M, Ananthakrishnan V, and Jaisri G. Stress as a Cause of Recurrent Aphthous Stomatitis and Its Correlation with Salivary Stress Markers. Chinese Journal of Physiology. 2017; 60 (4): 226-230.

29. Hossona Y \& Scully. Salivary changes in oral mucosal diseases. C. Periodontology 2000. 2016; 70: 111-127.

30. McCartan BE, Lamey PJ, Wallace AM. Salivary cortisol and anxiety in recurrent aphthous stomatitis. J Oral Pathol Med. 1996; 25:357-9.
31. Payne, L.A., Hibel, L.C., Granger, D.A., Tsao, J.C.I. and Zeltzer, L.K. Relationship of salivary alpha amylase and cortisol to social anxiety in healthy children undergoing laboratory pain tasks. J Child Adolesc. 2014;Behav. 2:1000129.

32. Sahu, G.K., Upadhyay, S. and Panna, S.M. Salivary alpha amylase activity in human beings of different age groups subjected to psychological stress. Indian J. Clin. Biochem. 2014; 29: 485- 490.

33. Rohleder, N., Wolf, J.M., Maldonado, E.F. and Kirschbaum, C. The psychosocial stress-induced increase in salivary alpha amylase is independent of saliva flow rate. Psychophysiology. 2006; 43: 645- 652.

34. Nater, U.M., Rohleder, N., Gaab, J., Berger, S., Jud, A., Kirschbaum, C. and Ehlert, U. Human salivary alphaamylase reactivity in a psychosocial stress paradigm. Int. J. Psychophysiol. 2005; 55:333- 342. 